Dynamic activity-travel diary data collection using a GPS-enabled personal digital assistant

Peer-reviewed author version

KOCHAN, Bruno; BELLEMANS, Tom; JANSSENS, Davy \& WETS, Geert (2006) Dynamic activity-travel diary data collection using a GPS-enabled personal digital assistant. In: Wang, Kelvin C. \& Smith, Brian etal (Ed.) Proceedings of the ninth International Conference on AATT. p. 319-324..

Handle: http://hdl.handle.net/1942/1371 


\title{
Dynamic activity-travel diary data collection using a GPS-enabled personal digital assistant
}

\author{
B. Kochan ${ }^{1}$, T. Bellemans ${ }^{1}$, D. Janssens ${ }^{1}$ and G. Wets ${ }^{1 *}$ \\ ${ }^{1}$ Transportation Research Institute (IMOB), Faculty of Applied Economics, Hasselt \\ University, Wetenschapspark 5 bus 6, B-3590 Diepenbeek, Belgium; PH (32) 1126 \\ 91 58; FAX (32) 11269199 ; email: \{Bruno.Kochan; Tom.Bellemans; \\ Davy.Janssens; Geert.Wets\}@UHasselt.be \\ ${ }^{*}$ Corresponding author
}

\begin{abstract}
Activity-based transportation models have set the standard for modelling travel demand for the last decade. It seems common practice nowadays to collect the data to estimate these activity-based transportation models by means of activitytravel diaries. This paper presents a general functional framework of an advanced activity-travel diary data collection application to be deployed on a GPS-enabled personal digital assistant (PDA). The different modules, which are the building blocks of the application, will be scrutinized as well.
\end{abstract}

\section{Introduction}

In the past, four-step models have been developed in order to predict travel demand in the long run. The predicted travel demand, as outcome of the four-step models, can be used to support different kinds of decisions such as e.g. investments in new road infrastructure. In these four-step models, travel is assumed to be the result of four subsequent decisions which are modelled separately. More recently, especially in the eighties and early nineties, several researchers claimed that very limited insight was offered into the relationship between travel and non-travel aspects in the widely adopted four-step models. Indeed, travel has an isolated existence in these models and the question why people undertake trips is completely neglected. This is where activity-based transportation models come into play. The major idea behind activity-based models is that travel demand is derived from the activities that individuals and households need or wish to perform. The main difference between traditional (i.e. four-step) transportation forecasting methodologies and activity-based transportation models is that the latter attempts to predict interdependencies between several facets of activity profiles. These facets are 
often identified as which activities are conducted where, when and for how long, with whom, and which transport modes are used.

As activity-based transportation models mature, they incorporate increasing levels of detail. An evolution towards dynamic activity-based models, which incorporates learning effects, can be observed in the literature (Joh 2004). The dynamics of travelbehaviour are driven by learning over time and short-term adaptation based on within-day rescheduling. As opposed to static models, dynamic models try to capture these dynamics using enhanced activity-travel data. In order to accommodate the growing data requirements for calibration and validation of the dynamic activitybased models, more detailed activity-travel diary data need to be collected. As the collection of basic activity-travel diary data puts a heavy burden on the respondents already, new techniques need to be developed to allow for the collection of even more detailed scheduling behaviour data. In this paper, a general functional framework of an advanced activity-travel diary data collection application to be deployed on a GPS enabled personal digital assistant (PDA) is presented. This tool must allow for the collection of detailed activity-travel diary data while limiting the burden for the respondents.

The remainder of this paper is organized as follows. The second section gives an overview of the state-of-the-art regarding computerized activity diary data collection tools. In the third section, the advantages and disadvantages of a GPS-enabled personal digital assistant will be discussed. In the fourth section, the functional description of the data collection tool will be given further consideration. In the last section, we will draw a conclusion.

\section{State-of-the-art in computerized activity-travel data collection tools}

CHASE (Computerized Household Activity Scheduling Elicitor) was the first computer aided self interview of activity scheduling behaviour (Doherty and Miller 2000). The purpose was to work out a survey which was able to track down the preceding scheduling process resulting into the definitive execution of an individual's schedule, along with the observed activity-travel patterns as the outcome. In the past, traditional survey techniques using diaries (e.g. paper-andpencil techniques) were limited almost exclusively to observed patterns, providing little insights into decision processes. This shortcoming was dealt with through the development of a multi-day computerized scheduling interface. The users' task consisted of keeping track of their scheduling decisions by adding, modifying and deleting activities to their schedule as they occurred, during a multi-day period. The application made notes of each of these scheduling decisions, along with prompting for additional information (e.g. the reasons for these decisions, the exact timing of these decisions). The latter would be much more complex using paper-and-pencil techniques. Initial testing results indicated that this computerized approach revealed a considerable amount of information on the scheduling process and observed patterns, whilst minimizing respondent burden (Doherty and Miller 2000). Many adjustments and sophistications of this method have followed the original approach, including 
applications on the Internet (Lee et al. 2000), development of a geographical information system interface for location and route tracking (Kreitz, Marion, Doherty and Rindsfüser 2002), and integration of GPS in a PDA application (Doherty et al. 2001).

In contributing to this line of research, the authors suggest an application which runs on a GPS enabled personal digital assistant (PDA). Several key development issues are: (i) desire to capture the dynamic activity-travel scheduling processes, (ii) desire to reduce respondent burden and (iii) to improve activity-travel data quality.

The application that is described in this paper captures the dynamic activity-travel scheduling process by first collecting information on the activities the respondent plans to execute and by collecting information on the activities that the respondent did execute in reality (diary) afterwards. Next, the planning and the diary are compared and additional information about the differences is gathered if required.

\section{Advantages and disadvantages of a GPS enabled personal digital assistant}

In the past, desktop computer-assisted data collection tools were used for filling out scheduling surveys which provided activity-travel diary data. However, these systems are not able to trace the actual activity-travel execution due to their mobility constraints. In order to solve this problem, one might think of a Personal Digital Assistant (PDA) with GPS technology for enhancing the data collection tool's mobility. The potential advantages of using a Personal Digital Assistant with GPS to supplement travel survey data collection are numerous: (i) when using a desktop computer-assisted data collection tool, the respondents have to remember the exact locations of their start and end positions, whereas with a PDA with GPS, trip origin, destination, and route data are automatically collected without burdening the respondent for the data; (ii) as the respondent may forget to report an activity trip, another advantage exists in recovery of unreported trips, as all routes are recorded; (iii) accurate trip start and end times are automatically determined, as well as trip lengths; (iv) the GPS data can be used to verify reported data; (v) both the data entry cost and the cost of post-processing the data, constitute a significant share of the total data collection cost (Zhou 2003). Both costs can be reduced to a minimum with computer-assisted forms of data collection.

One of the most important shortcomings of GPS technology is the fact that the system is not always reliable throughout the entire trip-recording period. Indeed, civilian GPS receivers have potential position errors resulting from e.g. multipath, selective availability,... However, by combining the GPS data with other data sources such as e.g. location information reported by the respondent and geographical information system (GIS) maps, these errors can in general be overcome. Another issue associated with the use of a hand-held device is the storage capacity available to save the data that is collected. However, with the ever decreasing storage capacity prices, PDA's can readily be fitted with sufficient memory to conduct the surveys at a reasonable price. As a PDA is powered by a 
battery, it has to be recharged regularly, which is an extra burden for the respondent. In order to reduce the number of times the PDA needs to be recharged, an energy conserving battery management system was integrated into the data collection application. This way the autonomy of the data collection tool can significantly be improved.

In the next section the functional description of the GPS-enabled activity-travel diary data collection tool is outlined.

\section{Functional description of the GPS-enabled activity-travel diary data collection tool}

The central theme of the data collection tool revolves around a Personal Digital Assistant (PDA). Compared to a typical computerized activity-travel diary data collection system (e.g. CHASE) a mobile system does not restrict the location for data collection and is easy to carry around by survey respondents for in-situation data input. Moreover, as the PDA is equipped with a GPS receiver, GPS data can be collected as well.

The system conceptually consists of two Graphical User Interfaces (GUI) (Household Survey and Activity Based Survey), a GPS logger, a data structure (Activity Diary \& Household Data and GPS Data), a data quality control module (Data Integrity Checks), a Trip Identification module, a GIS module and a Communication module (see Figure 1). The modular structure of the application allows for customization. The implementation of modules that are less important for the research at hand can be omitted.

The GPS logger is used to trace the physical travel paths and the travel times. If the GPS logger is active, it receives the GPS data from the GPS chip and stores it using the GPS Data module. The GPS logger collects data continuously and therefore it needs to operate in the background. This automatic feature has two advantages. In the first place, it facilitates data capturing and secondly, although the survey respondent may forget to register a new activity, the GPS logger captures the user's position during the travel period. This way, the system can prevent the loss of activity-travel data. Indeed, once the system detects a change in location that is not reported as travel by the survey respondent, the system prompts the respondent for additional information.

The GPS data, stored in the GPS Data module, can also be used for trip identification. Once the performed trip is identified, it can be used to verify whether the information about activities, reported by the respondent, is consistent with the actually recorded trip. In case there are inconsistencies, the respondent will be prompted for further clarification.

The Household Survey GUI inquires for personal demographic and activity/travelrelated information. These data are collected at the beginning of the survey period and are stored in the Activity Diary \& Household Data module. 
During the survey period, the respondent interacts frequently with the Activity Based Survey GUI, which is the major interface of the application. This GUI is used to register the activity-travel diary data during the survey period. It is used to enter, to modify or to delete an activity or a trip but it is also triggered if the Data Integrity Checks module detects an inconsistency (e.g. a city name that does not exist) and the Activity Diary Data needs to be altered.

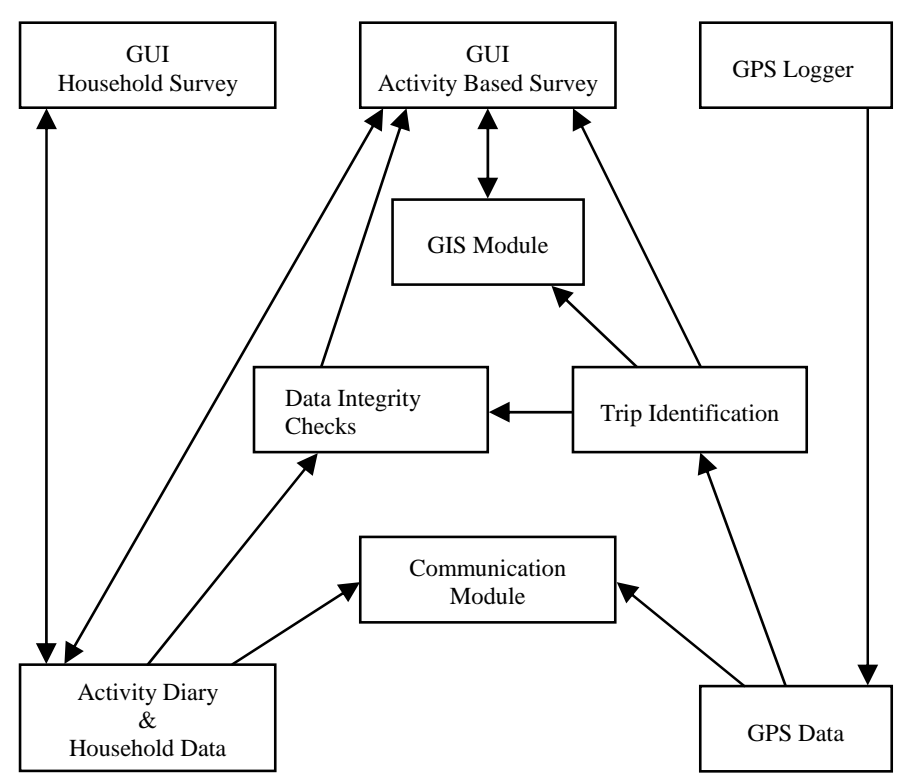

Figure 1. Schematic representation of the functional description modules composing the activity-travel diary data collection tool. The arrows denote the flow of information between modules.

The information stored on the hand-held devices can be downloaded through the Communication module. Depending on the implementation of the Communication module and on the available hardware, the data can be collected and stored on a data server either during or after the survey period.

The spatial dimension or the "where"-facet is the most difficult to collect in traditional paper-and-pencil diaries. People often do not precisely recall the exact location or the street name where a particular activity was carried out. Hence, traditional diaries are often restricted by limitations about the detail of information that is collected. The computer-assisted data collection tools can make a significant contribution here by integrating a geographical information system (GIS) module, which enables the user to either pinpoint a location on a map or to manually enter a location.

Computer-assisted data collection tools allow for data quality control. Indeed, a computer system can check for anomalies and prompt the respondent for additional 
information. Errors that report activities where the start hour of an activity is later than the end hour, activity locations that do not exist and many others are detected by the Data Integrity Checks module of the PDA application.

\section{Conclusion}

In this paper, a data collection tool, which is able to capture dynamic activitytravel scheduling behaviour was presented. The detailed data collected by this tool will be used to develop a dynamic activity-based transport model.

In the functional description of the application, a modular approach towards a general data collection application was presented. Next, the importance of each of these modules was described. Currently, the presented data collection tool is deployed in a large scale activity-travel survey in Flanders, Belgium.

\section{References}

Doherty, S.T., and Miller, E.J. (2000) A computerized household activity scheduling survey. Transportation, 27, (1), 75-97.

Doherty, S.T. et al. (2001) Moving beyond Observed Outcomes: Integrating Global Positioning Systems and Interactive Computer-Based Travel Behaviour Surveys. Transportation Research Circular, Transportation Research Board, National Research Council, Washington D.C., No. E-C026, 449-466.

Joh, C.H. (2004) Measuring and predicting adaptation in multidimensional activitytravel patterns. CIP-Data Koninklijke Bibliotheek, Den Haag, The Netherlands, ISBN 90-6814-577-0.

Kreitz, Marion, Doherty, S.T., and Rindsfüser, G. (2002) Collection of Spatial Behavioural Data and their Use in Activity Scheduling Models. Paper, 81st Annual Meeting of the Transportation Research Board, January 13-17, Washington D.C.

Lee, M.S. et al. (2000) Conducting an Interactive Survey of Household Weekly Activities via Internet: Preliminary Results from a Pilot Study. In Hensher, D. (Ed.), Proceedings of the 9th International Association for Travel Behaviour Research Conference, Gold Coast, Queensland, Australia July 2-7.

Zhou, J. (2003) Real-time Tracking of Activity Scheduling/Schedule Execution Within Unified Data Collection Framework. Proceedings of the 83rd Annual Meeting of the Transportation Research Board, July 2003 